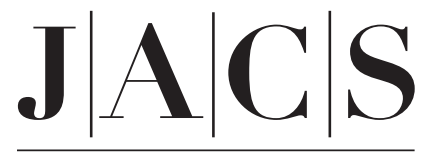

A R T I C L E S

Published on Web 09/17/2003

\title{
Solid-State Supramolecular Organization, Established Directly from Powder Diffraction Data, and Photoluminescence Efficiency of Rigid-Core Oligothiophene-S,S-dioxides
}

\author{
Emilio Tedesco, ${ }^{\dagger}$ Fabio Della Sala, ${ }^{\S}$ Laura Favaretto, ${ }^{\ddagger}$ Giovanna Barbarella, ${ }^{*, \ddagger}$ \\ David Albesa-Jove,,$^{\dagger}$ Dario Pisignano, $§$ Giuseppe Gigli,§ Roberto Cingolani,§ and \\ Kenneth D. M. Harris*,† \\ Contribution from the School of Chemical Sciences, University of Birmingham, \\ Edgbaston, Birmingham B15 2TT, United Kingdom, the National Nanotechnology Laboratories \\ of INFM and Dip. Ingegneria Innovazione, Universitá di Lecce, Via Arnesano, \\ 73100 Lecce, Italy, and Consiglio Nazionale Ricerche (ISOF), Via Gobetti 101, \\ I-40129 Bologna, Italy
}

Received April 10, 2003; E-mail: barbarella@isof.cnr.it; K.D.M.Harris@bham.ac.uk

\begin{abstract}
The "rigid-core" material 3,5-dimethyl-2,3'-bis(3-methylthiophene)-dithieno[3,2-b:',3'-d]thiophene4,4-dioxide (DTTOMe4) has the highest photoluminescence ever reported for thiophene-based molecules in the solid state. We report the structure of this material, determined directly from powder X-ray diffraction data using the Genetic Algorithm method for structure solution, followed by Rietveld refinement, and the structural properties are discussed in relation to the structures of the corresponding subsystems DTTO and DTTOMe. While the crystal structures of the latter compounds contain cofacial dimers, the crystal structure of DTTOMe4 comprises layers of molecules aligned in an antiparallel fashion. Intermediate neglect of differential overlap with single configuration interaction (INDO/SCl) calculations on the intermolecular interactions in the three crystal structures show that the different solid-state photoluminescence efficiencies of DTTOMe4, DTTOMe, and DTTO cannot be correlated with the different types of dipole-dipole alignment in the solid state. Instead, photoluminescence efficiencies correlate well with the rate of formation of nonradiatively decaying charge-transfer pairs upon photoexcitation. Because of larger intermolecular distances in DTTOMe4, the photoluminescence is less effectively quenched by charge-transfer processes than in DTTOMe and DTTO.
\end{abstract}

\section{Introduction}

Over the past few years, thiophene-based oligomers have generated considerable attention on account of their electrical and optical properties and their technological applications in optical and electronic devices, such as light-emitting diodes, ${ }^{1}$ lasers, ${ }^{2}$ field effect transistors,${ }^{3}$ and photovoltaic cells. ${ }^{4}$ Chemical modification of thienyl sulfur to the corresponding thienyl- $S, S$ dioxide leads to increased electron affinity ${ }^{5 a}$ and increased photoluminescence (PL) efficiency in the solid state, ${ }^{5 \mathrm{~b}}$ making oligothiophene- $S, S$-dioxides much better materials than con-

$\dagger$ University of Birmingham.

$\S$ Universitá di Lecce.

Consiglio Nazionale Ricerche

(1) (a) Gigli, G.; Barbarella, G.; Favaretto, L.; Cacialli, F.; Cingolani, R. Appl. Phys. Lett. 1999, 75, 439. (b) Gigli, G.; Inganas, O.; Anni, M.; De Vittorio, M.; Cingolani, R.; Barbarella, G.; Favaretto, L. Appl. Phys. Lett. 2001, 78, 1493.

(2) (a) Zavelani-Rossi, M.; Lanzani, G.; De Silvestri, S.; Anni, M.; Gigli, G.; Cingolani, R.; Barbarella, G.; Favaretto, L. Appl. Phys. Lett. 2001, 79, 4082. (b) Pisignano, D.; Anni, M.; Gigli, G.; Cingolani, R.; Zavelani-Rossi, M.; Lanzani, G.; Barbarella, G.; Favaretto, L. Appl. Phys. Lett. 2002, 81, 3534

(3) (a) Garnier, F. Acc. Chem. Res. 1999, 32, 209. (b) Dimitrakopoulos, C. D.; Malenfant, P. R. L. Adv. Mater. 2002, 14, 99.

(4) (a) Winder, C.; Muhlbacher, D.; Neugebauer, H.; Sariciftci, N. S.; Brabec, C.; Janssen, R. A. J.; Hummelen, J. K. Mol. Cryst. Liq. Cryst. 2002, 385, 213. (b) van Duren, J. K. J.; Loos, J.; Morrissey, F.; Leewis, C. M.; Kivits, K. P. H.; van Ijzendoorn, L. J.; Rispens, M. T.; Hummelen, J. C.; Janssen, R. A. J. Adv. Funct. Mater. 2002, 12, 665.

10.1021/ja035570o CCC: $\$ 25.00$ @ 2003 American Chemical Society ventional oligothiophenes for thin film electroluminescent diodes $^{1}$ and lasers. ${ }^{2}$

However, a serious difficulty with regard to improving thiophene-based organic devices is that there is insufficient knowledge about the optical and electrical properties of the materials in the solid state (i.e., single crystals, powders, and thin films). The optical and electrical properties of thiophene oligomers depend on molecular conformation and supramolecular organization in the solid state. In turn, molecular conformation and supramolecular organization are the result of the balance between the intrinsic molecular properties of the material and a variety of intramolecular and intermolecular interactions ${ }^{6,7}$ which depend on oligomer size, functionalization type, and substitution pattern. ${ }^{7,8}$ Substituents that may be used to improve solubility and processability can cause backbone distortions which affect the $\pi-\pi$ conjugation between adjacent thiophene rings and, hence, affect their electronic properties. Moreover, even in the presence of bulky substituents, ${ }^{5, c c}$ the

(5) (a) Barbarella, G.; Favaretto, L.; Zambianchi, M.; Pudova, O.; Arbizzani, C.; Bongini, A.; Mastragostino, M. Adv. Mater. 1998, 10, 551. (b) Bongini, A.; Barbarella, G.; Favaretto, L.; Sotgiu, G.; Zambianchi, M.; Casarini, D. Tetrahedron 2002, 58, 10151 .

(6) Gavezzotti, A.; Filippini, G. Synth. Met. 1991, 40, 255

(7) Marseglia, E. A.; Grepioni, F.; Tedesco, E.; Braga, D. Mol. Cryst. Liq. Cryst. 2000, 348, 137. 
energy barriers to rotation around the carbon-carbon bonds linking adjacent thiophene rings can be very low, leading to considerable conformational variability, even in the solid state. ${ }^{8 a}$ The situation is also complicated by the existence of polymorphs with different conformations, different crystal structures, and different optical properties. ${ }^{8 \mathrm{~d}}$

Because of the lack of well-established theoretical approaches for predicting the solid-state organization of organic molecules, ${ }^{9,10}$ the only viable way toward the control and optimization of devices based on molecular materials is through a trial and error procedure aimed at correlating a given type of molecular structure to specific self-assembly modalities in the solid state and further correlating these structural aspects with specific optical and electrical characteristics measured for single crystals, powders, and thin films.

With regard to oligothiophene- $S, S$-dioxides, comparison between the properties of single crystals obtained from a homogeneous series of trimer, pentamer, and heptamer allowed an empirical correlation between the photoluminescence efficiency and the structural characteristics of these materials to be established. ${ }^{8 \mathrm{c}}$ However, more recently, investigations aimed at establishing correlations between the molecular structure, the self-organization modes in the solid state, and the optical properties of thiophene oligomers on a more quantitative basis have been started ${ }^{11}$ by combining an understanding of spectroscopic data, crystal structures determined from diffraction data, and results from quantum chemistry calculations. Central to this type of investigation is knowledge of the solid-state conformation and crystal-packing modes of the photoluminescent and electroluminescent oligomers under investigation. Previously, ${ }^{11}$ single-crystal X-ray diffraction studies on a few quaterthiophenes and sexithiophenes have allowed the emission wavelengths, photoluminescence decay times, and photoluminescence efficiencies to be correlated to the degree of molecular distortion.

Unfortunately, most oligothiophenes are obtained in the form of microcrystalline powders for which structure determination using conventional single-crystal X-ray diffraction data is not feasible. As a consequence, for many of the thiophene-based oligomers that have been described in the literature, the crystal structures remain unknown. In recent years, however, considerable advances have been made in the power and scope of techniques for determining the structures of molecular crystals directly from powder diffraction data, thus providing the opportunity to determine structural information in those cases, such as the material DTTOMe4 studied here, that are not amenable to investigation by single-crystal diffraction methods. Recently, we have successfully employed these techniques ${ }^{12}$ to

(8) (a) Barbarella, G.; Zambianchi, M.; Bongini, A.; Antolini, L. Adv. Mater. 1993, 5, 834. (b) Barbarella, G.; Zambianchi, M.; Antolini, L.; Ostoja, P.; Maccagni, P.; Bongini, A.; Marseglia, E. A.; Tedesco, E.; Gigli, G.; Cingolani, R. J. Am. Chem. Soc. 1999, 121, 8920. (c) Antolini, L.; Tedesco, E.; Barbarella, G.; Favaretto, L.; Sotgiu, G.; Zambianchi, M.; Casarini, D.; Gigli, G.; Cingolani, R. J. Am. Chem. Soc. 2000, 122, 9006. (d) Gigli, G.; Lomascolo, M.; Cingolani, R.; Barbarella, G.; Zambianchi, M.; Antolini, L.; Della Sala, F.; Di Carlo, A.; Lugli, P. Appl. Phys. Lett. 1998, 73, 2414. (e) Barbarella, G.; Favaretto, L.; Sotgiu, G.; Antolini, L.; Gigli, G.; Cingolani, R.; Bongini, A. Chem. Mater. 2001, 13, 4112.

(9) (a) Desiraju, G. R. Crystal Engineering: The Design of Organic Solids; Elsevier: Amsterdam, 1989. (b) Desiraju, G. R.; Steiner, T. The Weak Hydrogen Bond in Structural Chemistry and Biology; Oxford University Press: New York, 1999.

(10) Gavezzotti, A. Crystallogr. Rev. 1998, 7, 5

(11) Gigli, G.; Della Sala, F.; Lomascolo, M.; Anni, M.; Barbarella, G.; Di Carlo, A.; Lugli, P.; Cingolani, R. Phys. Rev. Lett. 2001, 86, 167.
Scheme 1

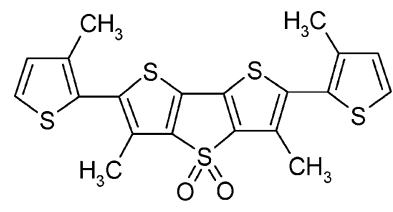

DTTOMe4

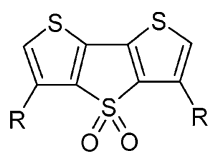

$\begin{array}{ll}\text { DTTOMe } & \mathrm{R}=\mathrm{Me} \\ \text { DTTO } & \mathrm{R}=\mathrm{H}\end{array}$ determine the crystal structures of a series of blue fluorescent thiophene-S,S-dioxides ${ }^{13}$ directly from powder X-ray diffraction data.

In this paper, we report the crystal structure of 3,5-dimethyl2,3'-bis(3-methylthiophene)-dithieno[3,2-b;2',3'-d] thiophene4,4-dioxide (DTTOMe4; Scheme 1), determined directly from powder X-ray diffraction data using the Genetic Algorithm (GA) technique for structure solution, followed by Rietveld refinement.

This structural information has been used together with semiempirical intermediate neglect of differential overlap with single configuration interaction (INDO/SCI) theoretical investigations on the photoluminescence properties of this material and on the related materials DTTOMe and DTTO (Scheme 1). The work is aimed at obtaining a semiquantitative correlation between the structural characteristics and the main intermolecular factors that are known to affect the solid-state photoluminescence of organic molecules. ${ }^{14}$

The reason for selecting rigid-core oligothiophene $S, S$ dioxides is that molecules of this type are characterized by high photoluminescence efficiencies both in solution and in the solid state, contrary to conventional thiophene oligomers. ${ }^{8 \mathrm{e}} \mathrm{DT}$ TOMe4 has the highest photoluminescence ever reported for thiophene-based molecules in the solid state and is an electroluminescent material. ${ }^{8 \mathrm{e}}$

We show that the strategy employed in this work accounts for the trend observed in the solid-state photoluminescence efficiencies of the three compounds and provides some clues with regard to engineering improved thiophene-based photoluminescent and electroluminescent materials.

Background to Structure Determination from Powder Diffraction Data. Among recent developments in techniques for solving crystal structures directly from powder diffraction data, the "direct-space" strategy ${ }^{15}$ is particularly suitable in the case of molecular materials. In this strategy, trial structures are generated in direct space, with the quality of each trial structure assessed by direct comparison between the powder diffraction pattern calculated for the trial structure and the experimental powder diffraction pattern (in our work, this comparison is made using the powder profile $R$-factor $R_{\text {wp }}$, which implicitly takes peak overlap into consideration). In the present paper, directspace structure solution has been carried out using our GA method $^{16-19}$ to locate the trial structure corresponding to the

(12) Harris, K. D. M.; Johnston, R. L.; Kariuki, B. M. Acta Crystallogr. 1998, A54, 632

(13) Tedesco, E.; Kariuki, B. M.; Harris, K. D. M.; Johnston, R. L.; Pudova, O.; Barbarella, G.; Marseglia, E. A.; Gigli, G.; Cingolani, R. J. Solid State Chem. 2001, 161, 121.

(14) Cornil, J.; Beljonne, D.; Calbert, J. P.; Brédas, J. L. Adv. Mater. 2001, 13, 1053 and references therein.

(15) Harris, K. D. M.; Tremayne, M.; Lightfoot, P.; Bruce, P. G. J. Am. Chem. Soc. 1994, 116, 3543 
global minimum in $R_{\mathrm{wp}}$. In this method, a population of trial structures is allowed to evolve subject to rules and operations (mating, mutation, and natural selection) analogous to those that govern evolution in biological systems. Each structure in the population is specified by its "genetic code", which represents, for each molecule in the asymmetric unit, the position $\{x, y, z\}$ and orientation $\{\theta, \phi, \psi\}$ of the molecule and the molecular conformation (defined by variable torsion angles $\{\tau 1, \tau 2, \ldots$, $\tau \mathrm{n}\}$ ). The quality ("fitness") of each structure in the population is assessed from its value of $R_{\mathrm{wp}}$. New structures are generated by the mating and mutation operations, and in our implementation used here, each new structure is subjected to local minimization of $R_{\mathrm{wp}}$. In the natural selection procedure, only the structures of highest fitness (i.e., lowest $R_{\mathrm{wp}}$ ) are allowed to pass from one generation to the next generation. In the GA calculation, the population is allowed to evolve for a specified number of generations or until convergence is reached. The best structure solution obtained in the GA structure solution calculation is used as the starting structural model for Rietveld refinement.

\section{Results}

Crystal Structure Determination of DTTOMe4 from Powder X-ray Diffraction Data. As discussed in the Experimental Section, determination of the unit cell and space group of DTTOMe4 was carried out straightforwardly from the powder $\mathrm{X}$-ray diffraction data. Structure solution was then carried out using our GA program EAGER. ${ }^{20}$ All non-hydrogen atoms were included in the structural model, and bond lengths and angles were set to standard values. With one molecule in the asymmetric unit (see Experimental Section), each structure was defined by a total of eight variables, $\left\{x, y, z, \theta, \varphi, \psi, \tau_{1}, \tau_{2}\right\}$, with the two torsion angles defining the orientations of the planes of the outer thiophene rings relative to the central ring system. The structure solution calculation involved the evolution of a population of 100 structures in 10 generations. In each generation, 100 offspring (50 pairs of parents) and 20 mutations were generated. In the mating operation, the eight variables were divided into four blocks $\{x, y, z\},\{\theta, \phi, \psi\},\left\{\tau_{1}\right\}$, and $\left\{\tau_{2}\right\}$. In the mating procedure between two selected parents, two of these blocks were chosen at random and exchanged between the two parents to generate the two offspring. The best structure solution $\left(R_{\mathrm{wp}}=7.36 \%\right)$, found after six generations, was taken as the starting structural model for Rietveld refinement, ${ }^{21}$ which was carried out using the GSAS program package. ${ }^{22}$ All atoms (with hydrogen atoms included into the structural model in calculated positions) were included in the refinement, with standard geometric restraints applied to bond lengths and bond angles.

(16) Kariuki, B. M.; Serrano-González, H.; Johnston, R. L.; Harris, K. D. M. Chem. Phys. Lett. 1997, 280, 189.

(17) Harris, K. D. M.; Johnston, R. L.; Kariuki, B. M. Acta Crystallogr. 1998, A54, 632 .

(18) Turner, G. W.; Tedesco, E.; Harris, K. D. M.; Johnston, R. L.; Kariuki, B. M. Chem. Phys. Lett. 2000, 321, 183.

(19) Tedesco, E.; Turner, G. W.; Harris, K. D. M.; Johnston, R. L.; Kariuki, B M. Angew. Chem., Int. Ed. 2000, 39, 4488.

(20) Harris, K. D. M.; Johnston, R. L.; Albesa Jové, D.; Chao, M. H.; Cheung, E. Y.; Habershon, S.; Kariuki, B. M.; Lanning, O. J.; Tedesco, E.; Turner, G. W. Evolutionary Algorithm Generalized for Energy and R-factor University of Birmingham, Birmingham, U.K., 2001 [an extended version of the program GAPSS, Harris, K. D. M.; Johnston, R. L.; Kariuki, B. M.; University of Birmingham, Birmingham, U.K., 1997].

(21) Rietveld, H. M. J. Appl. Crystallogr. 1969, 2, 65.

(22) Larson, A. C.; Von Dreele, R. B. Report No. LA-UR-86-748; Los Alamos National Laboratory, Los Alamos, NM, 1987.

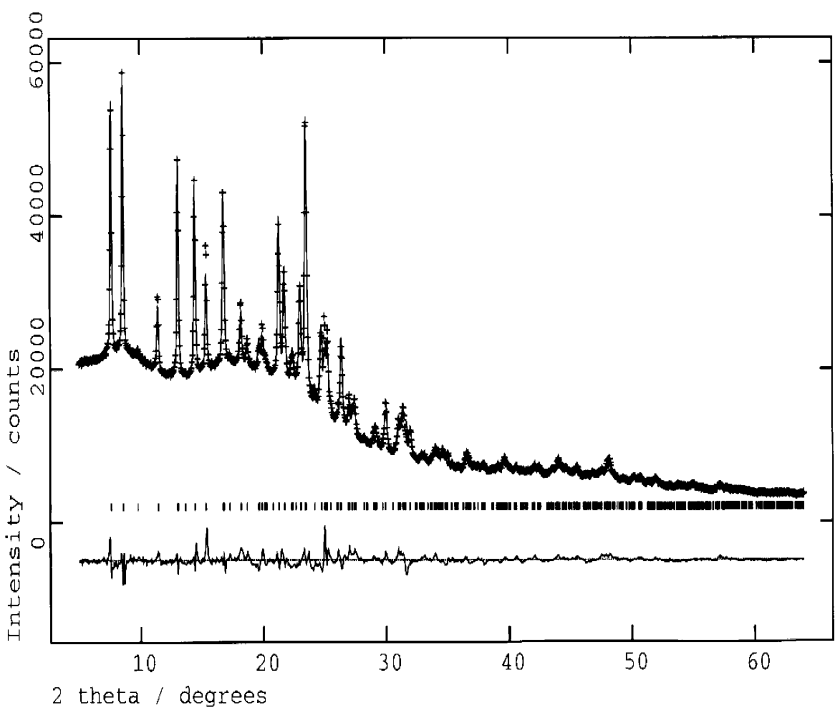

Figure 1. Experimental ( + marks), calculated (solid line), and difference (bottom) powder diffraction profiles for the Rietveld refinement of DTTOMe4 $\left(R_{\mathrm{wp}}=3.48 \% ; R_{\mathrm{p}}=2.50 \%\right)$. Reflection positions are marked. The calculated powder diffraction profile is for the final refined crystal structure.

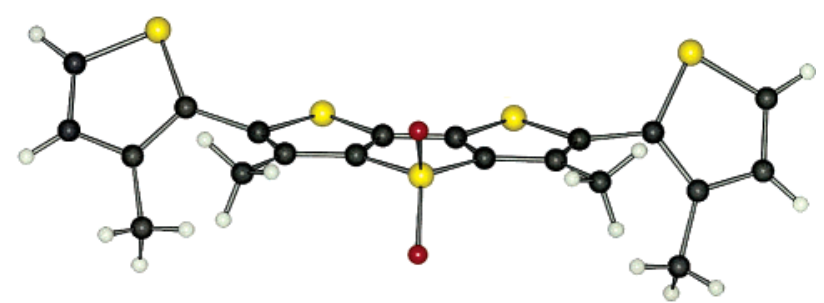

Figure 2. Molecular geometry of DTTOMe4 in the solid state showing the syn arrangement of the outer thiophene rings.

A rigid body refinement strategy was adopted in this case, with each of the ring systems maintained as strictly planar units. Two common isotropic displacement parameters were refined for non-hydrogen atoms belonging to the central rigid core unit $\left(U_{\text {iso }}=0.076 \AA^{2}\right)$ and for atoms of the outer thienyl moieties $\left(U_{\text {iso }}=0.094 \AA^{2}\right)$. In the final stages, a preferred orientation parameter was refined. The final Rietveld refinement (total number of variables, 33; number of reflections, 1434; number of data points, 3089) gave agreement indices $R_{\mathrm{wp}}=3.48 \%$ and $R_{\mathrm{p}}=2.50 \%$ (see Figure 1). Fractional coordinates in the final refined structure are given in Table 1.

The results show that the conformation of DTTOMe4 in the crystal structure is significantly nonplanar, with the outer thiophene rings in a noncoplanar syn arrangement with respect to the central unit, with torsion angles of 60.7 and $57.4^{\circ}$ (see Figure 2). This conformation is similar to that found in single crystals of head-to-head methyl-substituted quaterthiophene and sexithiophene derivatives. ${ }^{8 \mathrm{a}, \mathrm{b}}$ The crystal structure of DTTOMe4 can be described in terms of layers of molecules in the $a c$ plane aligned in an antiparallel fashion along the [101] direction, as illustrated in Figure 3.

Table 2 compares the short intermolecular contacts and packing coefficient in the crystal structure of DTTOMe4 with those in the crystal structures of DTTOMe and DTTO, which have been determined previously ${ }^{8 e}$ from single-crystal X-ray diffraction data. 
Table 1. Fractional Coordinates and Isotropic Displacement Parameters for the Non-Hydrogen Atoms in the Final Refined Crystal Structure of DTTOMe4a

\begin{tabular}{cccccrrr}
\hline atom & $x$ & $y$ & $z$ & atom & $x$ & \multicolumn{1}{c}{$z$} \\
\hline $\mathrm{S}(1)$ & $0.462(2)$ & $0.003(1)$ & $0.2206(9)$ & $\mathrm{C}(15)$ & $0.7247(4)$ & $0.101(2)$ & $0.1030(7)$ \\
$\mathrm{C}(2)$ & $0.531(2)$ & $0.0975(9)$ & $0.3042(5)$ & $\mathrm{O}(16)$ & $0.8156(6)$ & $0.060(2)$ & $0.3966(9)$ \\
$\mathrm{C}(3)$ & $0.5183(1)$ & $0.2149(7)$ & $0.3772(4)$ & $\mathrm{O}(17)$ & $0.7868(7)$ & $-0.205(1)$ & $0.307(2)$ \\
$\mathrm{C}(4)$ & $0.4535(2)$ & $0.213(2)$ & $0.376(1)$ & $\mathrm{S}(18)$ & $0.7243(3)$ & $0.129(2)$ & $-0.0376(4)$ \\
$\mathrm{C}(5)$ & $0.4173(1)$ & $0.115(2)$ & $0.290(1)$ & $\mathrm{C}(19)$ & $0.8062(2)$ & $0.084(1)$ & $-0.0048(3)$ \\
$\mathrm{C}(6)$ & $0.5694(2)$ & $0.3005(4)$ & $0.4706(3)$ & $\mathrm{C}(20)$ & $0.8327(2)$ & $0.0352(8)$ & $0.1079(3)$ \\
$\mathrm{C}(7)$ & $0.5929(2)$ & $0.091(1)$ & $0.2700(4)$ & $\mathrm{C}(21)$ & $0.8983(2)$ & $-0.0394(4)$ & $0.1544(3)$ \\
$\mathrm{S}(8)$ & $0.5931(2)$ & $0.129(2)$ & $0.1286(5)$ & $\mathrm{C}(22)$ & $0.8391(3)$ & $0.066(1)$ & $-0.0983(3)$ \\
$\mathrm{C}(9)$ & $0.6754(3)$ & $0.098(2)$ & $0.1652(7)$ & $\mathrm{S}(23)$ & $0.7993(3)$ & $-0.021(1)$ & $-0.2283(4)$ \\
$\mathrm{C}(10)$ & $0.6941(3)$ & $0.014(2)$ & $0.2676(6)$ & $\mathrm{C}(24)$ & $0.8582(6)$ & $0.057(2)$ & $-0.2849(7)$ \\
$\mathrm{C}(11)$ & $0.6506(2)$ & $0.0287(8)$ & $0.3373(3)$ & $\mathrm{C}(25)$ & $0.9046(4)$ & $0.148(2)$ & $-0.2086(5)$ \\
$\mathrm{C}(12)$ & $0.6653(2)$ & $-0.0200(4)$ & $0.4614(3)$ & $\mathrm{C}(26)$ & $0.8956(2)$ & $0.1482(8)$ & $-0.0992(2)$ \\
$\mathrm{S}(13)$ & $0.7778(2)$ & $-0.0338(9)$ & $0.3010(4)$ & $\mathrm{C}(27)$ & $0.9465(2)$ & $0.2059(4)$ & $0.0065(3)$ \\
$\mathrm{C}(14)$ & $0.7833(3)$ & $0.038(3)$ & $0.1662(7)$ & & & & \\
\hline
\end{tabular}

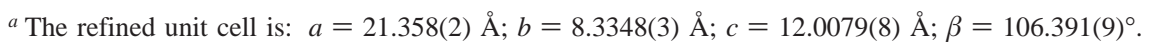

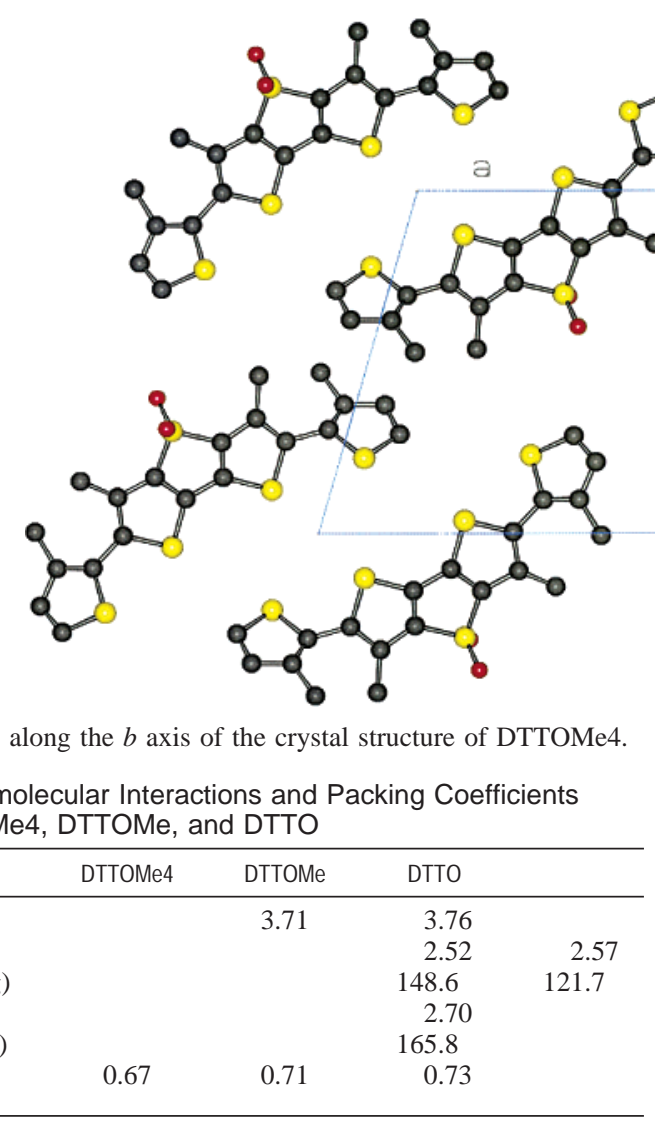

The crystal structures of both DTTOMe and DTTO contain sandwiched "dimers", with $\pi \cdots \pi$ distances of 3.76 and $3.71 \AA$, respectively. ${ }^{8 \mathrm{e}}$ In DTTO, the three-dimensional organization is achieved through $\pi \cdots \pi$ stacking, ${ }^{8}$ e in agreement with typical observations for fused aromatic systems. In DTTOMe, although the dimeric subunit is retained, there is also a "herringbonelike" motif involving short $\mathrm{C}-\mathrm{H} \cdots \pi$ hydrogen bonds $\left(\mathrm{H} \cdots \pi=2.70 \AA\right.$ 的 $\left.\mathrm{C}-\mathrm{H} \cdots \pi=165.8^{\circ}\right){ }^{8 \mathrm{e}}$

In contrast, the crystal structure of DTTOMe4 contains no recognizable "dimers", and no short intermolecular contacts of the type identified above for DTTOMe and DTTO are present. Consideration of packing coefficients (Table 2) suggests that the efficiency of volume occupation is slightly lower for DTTOMe4 than for DTTOMe and DTTO (packing coefficients $0.67,0.71$, and 0.73 , respectively).
Optical Properties of DTTOMe4, DTTOMe, and DTTOMe. The wavelengths $\lambda_{\max }$ and $\lambda_{\text {PL }}$ for DTTOMe4, DTTOMe, and DTTO in the solid state and in solution have been reported previously ${ }^{8 \mathrm{e}}$ and are in line with the trend generally observed for oligothiophene-S,S-dioxides. The photoluminescence spectra of microcrystalline powders of DTTOMe4, DTTOMe, and DTTO are shown in Figure 4. All spectra are broad and do not show well-defined vibronic features at room temperature.

Absolute photoluminescence efficiency measurements ( $\eta$, the number of photons re-emitted radiatively as a percentage of the number of photons absorbed, measured using an integrating sphere $^{23}$ ) were made on microcrystalline powders. The results confirm the experimental trend reported previously (see also Table 5). ${ }^{8 \mathrm{e}}$

Theoretical Calculations. Semiempirical quantum mechanics calculations have been carried out using the INDO/SCI ${ }^{24}$ method to compare the intermolecular interactions in the crystal structures of DTTOMe4, DTTOMe, and DTTO. Since clusters of several molecules need to be considered, and given the large size of the oligothiophene systems, ab initio methods are

(23) Greenham, N. C.; Samuel, I. D. W.; Hayes, G. R.; Phillips, R. T.; Kessener, Y. A. R. R.; Moratti, S. C.; Holmes, A. B.; Friend, R. H. Chem. Phys. Lett. 1995, 241, 89

(24) Ridley, J. E.; Zerner, M. C. Theor. Chim. Acta 1973, 32, 111. 


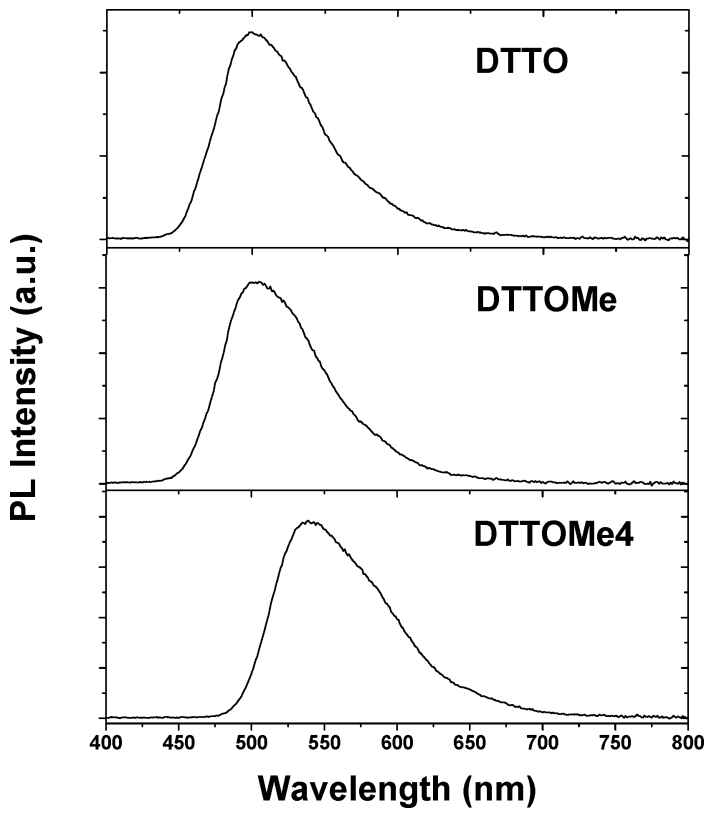

Figure 4. Photoluminescence spectra of the microcrystalline powders of DTTO, DTTOMe, and DTTOMe4.

Table 3. INDO/SCI $\mathrm{S}_{1}$ Calculated Excitation Energies ( $\left.E_{\text {calcd }}\right)$ and Oscillator Strengths $\left(f_{\text {osc }}\right)$, and Experimental Maximum Energy Values of the Absorption Spectra in Solution $\left(E_{\text {soln }}\right)$ and in the Solid-State $\left(E_{\mathrm{sol}}\right)$ of DTTO, DTTOMe, and DTTOMe4

\begin{tabular}{lcccc}
\hline & $E_{\text {calcod }} / \mathrm{eV}$ & $f_{\text {osc }}$ & $E_{\text {son }} / \mathrm{eV}$ & $E_{\text {sol }} / \mathrm{eV}$ \\
\hline DTTO & 3.310 & 0.4029 & 3.54 & 3.47 \\
DTTOMe & 3.277 & 0.3770 & 3.41 & 3.44 \\
DTTOMe4 & 2.733 & 0.7269 & 3.10 & 3.00 \\
\hline
\end{tabular}

prohibited by the high computational demand. Indeed, most theoretical investigations of such systems ${ }^{14,25}$ have been carried out using semiempirical methods.

First, we considered the properties of single molecules of DTTOMe4, DTTOMe, and DTTO, taking the molecular conformation in each case directly from the crystal structure. Then, the effects of intermolecular interactions were investigated by considering clusters of molecules taken from the crystal structures.

The energy of the first singlet excited state $\left(\mathrm{S}_{1}\right)$ and the oscillator strength of the optical transition are reported for DTTOMe4, DTTOMe, and DTTO in Table 3. The strongly allowed $\mathrm{S}_{1}$ state dominates the optical properties and is predominantly (about 90\%) characterized by a HOMO $\rightarrow$ LUMO transition. The calculated $\mathrm{S}_{1}$ energies can be compared to the maximum energies of the experimental absorption spectra, which are reported in the last two columns of Table 3. The agreement between calculated and experimental values is very good, both for solution state and solid-state measurements. This result indicates that, in the solid state, exciton resonance interactions are weak in all compounds.

The excitonic interactions $(\gamma)$ are proportional to the rate of excitation transfer between molecules. Thus, for a crystal with small $\gamma$ values, the optical properties of the solid resemble those of single molecules, but for crystals with high $\gamma$ values, the nature of the crystal structure and the intermolecular interactions have a major influence on the optical properties. The $\gamma$ values

(25) Beljonne, D.; Cornil, J.; Silbey, R.; Milliè, P.; Brédas, J. L. J. Chem. Phys. 2000, 112, 4749 .
Table 4. INDO/SCI Calculated Exciton Resonance Interactions in DTTO, DTTOMe, and DTTOMe4 for the Two Molecular Pairs Forming the Highest $\mathrm{H}$-type $\left(h_{\max 1}, h_{\max 2}\right)$ and J-type $\left(j_{\max }\right)$ Aggregates

\begin{tabular}{lccc}
\hline & $\gamma\left(H_{\text {max }}\right) / \mathrm{eV}$ & $\gamma\left(H_{\max }\right) / \mathrm{eV}$ & $\gamma\left(J_{\max }\right) / \mathrm{eV}$ \\
\hline DTTO & 0.079 & 0.023 & -0.037 \\
DTTOMe & 0.078 & 0.030 & -0.021 \\
DTTOMe4 & 0.060 & 0.031 & -0.025 \\
\hline
\end{tabular}

Table 5. INDO/SCI Calculated Values of the Integrals $T_{\mathrm{e}}^{\max }, t_{\mathrm{e}}^{\mathrm{sum}}$, $t_{\mathrm{h}}^{\text {max }}$, and $t_{\mathrm{h}}^{\text {sum }}$ (see text for definitions) and Experimental Quantum Yield $(\eta)$ in DTTO, DTTOMe, and DTTOMe4 ${ }^{a}$

\begin{tabular}{lccccc}
\hline & $t_{\mathrm{e}}^{\max } / \mathrm{eV}$ & $f_{\mathrm{e}}^{\mathrm{sum}} / \mathrm{eV}$ & $t_{\mathrm{h}}^{\max } / \mathrm{eV}$ & $t_{\mathrm{h}}^{\mathrm{sum}} / \mathrm{eV}$ & $\eta / \%$ \\
\hline DTTO & 0.007 & 0.141 & 0.199 & 1.816 & $12 \%$ \\
DTTOMe & 0.031 & 0.210 & 0.210 & 1.052 & $16 \%$ \\
DTTOMe4 & 0.005 & 0.080 & 0.032 & 0.343 & $48 \%$ \\
\hline
\end{tabular}

a Values of $\eta$ are taken from ref $8 \mathrm{e}$.

in the solid state can be calculated using the INDO/SCI transition densities $P(\mathbf{r})$ of the $\mathrm{S}_{1}$ state, using the expression ${ }^{11}$

$$
\gamma_{\mathrm{AB}}=\iint \frac{P_{\mathrm{A}}(\mathbf{r}) P_{\mathrm{B}}\left(\mathbf{r}^{\prime}\right)}{\left|\mathbf{r}-\mathbf{r}^{\prime}\right|} \mathrm{d} \mathbf{r} \mathrm{d} \mathbf{r}^{\prime}
$$

We calculated the exact exciton resonance interaction, ${ }^{11}$ denoted $\gamma_{\mathrm{AB}}$, for each pair $(\mathrm{A}, \mathrm{B})$ of adjacent molecules within the crystal structure. In the crystals of DTTOMe4, DTTOMe, and DTTO, pairs of adjacent molecules form either $H$-type (co-facial configuration, $\gamma>0$ ) or $J$-type (staggered configuration, $\gamma<$ 0 ) aggregates, depending on the direction within the crystal. We note that our computed values $\gamma_{\mathrm{AB}}$ are exact, in the sense that they include all the multipole terms, whereas $\gamma_{\mathrm{AB}}$ is often computed within the dipole-dipole approximation, which strongly overestimates the result for near molecules. ${ }^{14,25}$ In Table 4 , we report the values of $\gamma_{\mathrm{AB}}$ for the three different crystals, focusing in each case on the two molecular pairs that form the strongest $H$ aggregates $\left(H_{\max 1}, H_{\max 2}\right)$ and the molecular pair that forms the strongest $J$ aggregate $\left(J_{\max }\right)$. The values of $\gamma$ shown in Table 4 are small and are about half those calculated for quaterthiophene and some of its derivatives. ${ }^{11}$

Finally, using the INDO/SCI wave functions, we calculated the electron and hole transfer integrals for each pair of molecules $\mathrm{A}$ and $\mathrm{B}$ in the crystal structure, as given by $t_{\mathrm{e}}[\mathrm{A}, \mathrm{B}]=$ $\left\langle\mathrm{HOMO}_{\mathrm{A}}|H| \mathrm{HOMO}_{\mathrm{B}}\right\rangle$ and $t_{\mathrm{h}}[\mathrm{A}, \mathrm{B}]=\left\langle\mathrm{LUMO}_{\mathrm{A}}|H| \mathrm{LUMO}_{\mathrm{B}}\right\rangle$, respectively. These integrals are related to the probability of formation of a charge-transfer pair $^{26}$ and are proportional to the electronic bandwidth in the $\mathrm{A}-\mathrm{B}$ direction. ${ }^{14}$

In Table 5, we report the maximum transfer integrals $\left(t_{\mathrm{e}}^{\max }\right.$ and $\left.t_{\mathrm{h}}^{\max }\right)$ among all molecular pairs in the crystal and the summations $\left(t_{\mathrm{e}}^{\text {sum }}=\Sigma_{\mathrm{AB}} t_{\mathrm{e}}[\mathrm{A}, \mathrm{B}]\right.$ and $\left.t_{\mathrm{h}}^{\text {sum }}=\Sigma_{\mathrm{AB}} t_{\mathrm{h}}[\mathrm{A}, \mathrm{B}]\right)$ over all molecular pairs (with $\mathrm{A}$ in the central unit cell). We note that since $t_{\mathrm{e}}[\mathrm{A}, \mathrm{B}]$ and $t_{\mathrm{h}}[\mathrm{A}, \mathrm{B}]$ decay exponentially with $\mathrm{A} \cdots \mathrm{B}$ distance, only first neighbors make a significant contribution to the summations $t_{\mathrm{e}}^{\text {sum }}$ and $t_{\mathrm{h}}^{\text {sum }}$. These summations represent averaged quantities for electron and hole transfer, respectively, along all directions. Table 5 shows that the values of $t_{\mathrm{h}}^{\max }$ for DTTO and DTTOMe are much larger than those calculated for quaterthiophene and its derivatives. ${ }^{11}$ Moreover, the values of

(26) Della Sala, F.; Heinze, H. H.; Görling, A. Chem. Phys. Lett. 2001, 339, 343. 
$t_{\mathrm{h}}^{\max }$ for DTTO and DTTOMe are an order of magnitude larger than that for DTTOMe4.

\section{Discussion}

The crystal structure of DTTOMe4 determined directly from powder diffraction data shows that the molecular conformation is significantly nonplanar, with torsion angles of 60.7 and $57.4^{\circ}$ between the ring systems (Figure 3 ). This conformation is very similar to that found for a tetramethyl quaterthiophene with the same regiochemistry of substitution, ${ }^{8 a}$ the unpredictable geometry of which, with external syn thiophene rings, was ascribed to a complex pattern of intramolecular and intermolecular interactions. ${ }^{7}$ The same geometry was also found for one of the polymorphs of a sexithiophene containing a head-to-head tetramethylated fragment. ${ }^{8 b}$

In contrast to the situation for DTTO and DTTOMe, no "dimers" are present in the crystal structure of DTTOMe4. The driving force for the formation of dimeric units in the crystal structures of DTTO and DTTOMe is the antiparallel dipolar arrangement of the $\mathrm{SO}_{2}$ groups in the central moiety. ${ }^{8 e}$ Apparently, the presence of the external methylated thiophene rings in DTTOMe4 prevents the molecules from forming close pairs, and hence, no short intermolecular contacts are found (Table 2).

Our INDO/SCI calculations show that for all compounds there is very good agreement between the $S_{1}$ singlet excitation energies (calculated using molecular geometries from the crystal structures) and the experimental maximum energy values of the absorption spectra in the solid state as well as in solution (Table 3). On one hand, this means that the INDO/SCI approximation reproduces the optical properties of this type of molecule well, and on the other hand, that exciton resonance interactions in the solid state (i.e., the interactions between the neutral excited states generated by photoexcitation) must be weak.

The calculated exciton resonance interactions $(\gamma$, which is proportional to the rate of excitation transfer between molecules) are indeed small and, more importantly, quite similar for DTTOMe4, DTTOMe, and DTTO (see Table 4). The value of $\gamma\left(H_{\max 1}\right)$ is somewhat larger for DTTO and DTTOMe than for DTTOMe4, but in the latter case the value of $\gamma\left(H_{\max 2}\right)$ is also comparable, which is not the situation for DTTO and DTTOMe. Furthermore, any relationship of $J$ aggregates with photoluminescence efficiency can be ruled out. Thus, the different photoluminescence efficiencies displayed by these compounds in the solid state cannot be explained by a different type of dipole alignment.

In solution, the photoluminescence efficiencies of DTTOMe4, DTTOMe, and DTTO are quite high and very similar to each other $^{8 \mathrm{e}}(85,77$, and $75 \%$, respectively), indicating that the electronic structure and intramolecular nonradiative channels do not differ significantly between these molecules.

In the solid state, photoluminescence quantum yields are 2-4 times smaller than in solution and are different for each of the DTTOMe4, DTTOMe, and DTTO molecules. Thus, since the molecular structure of such rigid compounds changes very little in the solid state compared to solution, the different photoluminescence efficiencies measured in the solid state should reflect different nonradiative effects due to different intermolecular interactions in the three crystal structures.

The role of charge-transfer excitons (when the $\pi$ electron is excited from one chain and promoted to an unoccupied level located on an adjacent chain) in photoluminescence quenching has been evaluated for the three compounds by calculating the interchain transfer integrals ${ }^{27,28}$ for each molecular pair in the crystal structures. The results reported in Table 5 show that there are marked differences in the probability of charge-transfer pair formation in DTTOMe4, DTTOMe, and DTTO. The electrontransfer integrals are found to be smaller than hole transfer integrals, such that the former can be neglected.

According to the data reported in Table 5, DTTOMe has the highest $t_{\mathrm{h}}^{\max }$, which may be attributed to the presence of strongly interacting molecular pairs (as discussed above; see Table 2). However, this material does not have the highest sum $t_{\mathrm{h}}^{\text {sum }}$ of all transfer integrals, as such dimers represent only a small fraction of all molecular pairs. Instead, for DTTO, $t_{\mathrm{h}}^{\max }$ is about $15 \%$ smaller than that for DTTOMe, but there is a much higher concentration of dimers and, as a consequence, the value of $t_{\mathrm{h}}^{\mathrm{sum}}$ is almost a factor of 2 larger than that for DTTOMe. For DTTOMe4, both $t_{\mathrm{h}}^{\max }$ and $t_{\mathrm{h}}^{\text {sum }}$ are substantially smaller than those for DTTO and DTTOMe because of the larger intermolecular distances.

Thus, the different quantum photoluminescence efficiencies of DTTOMe4, DTTOMe, and DTTO measured in the solid state $(48,16$, and $12 \%$, respectively) can be very well related to the $t_{\mathrm{h}}^{\text {sum }}$ values. Higher $t_{\mathrm{h}}^{\text {sum }}$ values mean higher rate of formation of charge-transfer pairs, which decay nonradiatively and thus lower the photoluminescence efficiency. As a consequence, the photoluminescence is more efficiently quenched in DTTO and DTTOMe than in DTTOMe4.

These results suggest that a similar mechanism might also explain the very different photoluminescence quantum yields measured for ter-, quinque-, and heptathiophene- $S, S$-dioxides in the solid state. ${ }^{8 \mathrm{c}}$ In an attempt to rationalize the experimental results, we ascribed the different photoluminescence efficiencies of these compounds $(45,12$, and $2 \%$, respectively) to the different orientations of the long molecular axes in the crystal structures. Single-crystal X-ray diffraction analysis showed indeed that the trimer crystallizes with marked tilting, whereas the heptamer adopts a strictly parallel alignment of the long molecular axes. The pentamer adopts an intermediate structural situation. However, the data reported in this work for DTTOMe4 show that, even when oligothiophene-S,S-dioxide molecules pack with their long molecular axes parallel, the photoluminescence efficiency can be quite high if the molecules are sufficiently distant with respect to one another. Thus, the qualitative criterion relying on the orientation of the molecular axes is not valid for oligothiophene- $S, S$-dioxides. A more effective criterion may devolve upon the trend concerning variation of packing coefficient within a structurally correlated set of molecules (our data show that the packing coefficients (Table 2) and the photoluminescence efficiencies of DTTO, DTTOMe, and DTTOMe4 correlate inversely), although we note both the inherent assumptions in the calculation of packing coefficients and the fact that there are only small differences between the values of packing coefficients for the three materials studied. The same trend also holds for the set of ter-, quinque-, and heptaoligothiophene- $S, S$-dioxides mentioned above. ${ }^{8 \mathrm{c}}$

(27) Wu, M. W.; Conwell, E. M. Phys. Rev B 1997, 56, R10060.

(28) Hennessy, M. H.; Soos, Z. G.; Pascal, R. A.; Girlando, A. Chem. Phys. 1999, 245, 1999. 
The behavior of the material DTTOMe4 investigated in this work is different from that found previously for a series of substituted quaterthiophenes (see ref 11), for which photoluminescence efficiency was found to be a molecular property, in that it may be understood on the basis of the molecular distortion induced by substituent effects and crystal packing. It is relevant to note that DTTO, DTTOMe, and DTTOMe4 are rigid molecules, whereas quaterthiophenes are very flexible.

\section{Concluding Remarks}

In the series of materials studied in this paper, for which the lumophore is the dithienothiophene-S,S-dioxide moiety, the intermolecular interactions play a major role in determining the solid-state photoluminescence efficiency. We have demonstrated that simple qualitative criteria are unable to rationalize the relationship between the molecular structure and the photoluminescence efficiency. Instead, a more sophisticated approach, relying on the combined knowledge of crystal structures, spectroscopic measurements and theoretical calculations, is required to secure a sound understanding of the observed trends. We emphasize that new opportunities for determining the crystal structures of molecular materials directly from powder diffraction data were essential for establishing the structural properties of one of the materials required for this study.

\section{Experimental Section}

Powder X-ray Diffraction. The powder X-ray diffraction pattern of 3,5-dimethyl-2,3'-bis(3-methylthiophene)-dithieno[3,2-b;2',3'-d]thiophene-4,4-dioxide (DTTOMe4, prepared according to the method described in ref 8e) was recorded in transmission mode on a Siemens D5000 diffractometer at ambient temperature using Ge-monochromated $\mathrm{Cu} \mathrm{K} \alpha_{1}$ radiation $(\lambda=1.5406 \AA)$ and a linear position-sensitive detector. The total $2 \theta$ range was $5-65^{\circ}$, measured in steps of $0.02^{\circ}$ over a total time of $12 \mathrm{~h}$. The powder diffraction pattern was indexed by the program DICVOL $^{29}$ to give the following unit cell with monoclinic metric symmetry: $a=21.469 \AA, b=8.389 \AA, c=12.083$ $\AA, \beta=106.24^{\circ}$. This unit cell gave a good Le Bail fit ${ }^{30}$ with an agreement factor $R_{\mathrm{wp}}=2.01 \%$. Density considerations suggest that there are four molecules in the unit cell, and consideration of systematic absences suggests that the space group is $P 2{ }_{1} / a$. For this space group and with four molecules in the unit cell, there is one molecule in the asymmetric unit.

The crystal structures of 2,3'-bis(3-methylthiophene)-dithieno[3,2$\left.\mathrm{b} ; 2^{\prime}, 3^{\prime}-\mathrm{d}\right]$ thiophene-4,4-dioxide (DTTOMe) and dithieno[3,2-b;2', $3^{\prime}-$ d] thiophene-4,4-dioxide (DTTO) determined from single-crystal X-ray diffraction are reported in ref $8 \mathrm{e}$.

Optical Measurements. The photoluminescence spectra and quantum efficiencies $(\eta)$ of DTTO, DTTOMe, and DTTOMe4 in the solid state were measured by placing the microcrystalline powders into a 4-in. integrating sphere (Labsphere) and by exciting them with a $\mathrm{He}-$ Cd laser $(\lambda=325 \mathrm{~nm})$ under a flux of purging nitrogen to inhibit photooxidation. The photoluminescence signal was collected by means of a CCD spectrograph (Ocean Optics).

Theoretical Calculations. All calculations were performed using the in-house-developed program MOCI, with the calculations run on a Compaq XP1000 workstation.

Acknowledgment. This article is dedicated in memory of Emilio Tedesco. We are deeply touched by the death of our young colleague and friend Emilio Tedesco, who was a nice person and a fine scientist. We thank Prof. Luciano Antolini (Universitá di Modena e Reggio Emilia) for his helpful assistance. We are grateful to EPSRC and Wyeth-Ayerst for financial support.

JA035570O

(29) Boultif, A.; Louër, D. J. Appl. Crystallogr. 1991, 24, 987.

(30) Le Bail, A.; Duroy, H.; Fourquet, J. L. Mater. Res. Bull. 1988, 23, 447. 